\title{
Nonpool based spectrum sharing for two UMTS operators in the UMTS Extension Band
}

\author{
Gbenga Salami, Atta Ul Quddus, Duminda Thilakawardana, Rahim Tafazolli \\ Mobile VCE Research Group, Center for Communication Systems Research, \\ University of Surrey, GU2 7XH, Guildford, \\ Surrey, United Kingdom. \\ \{G.Salami, A.Quddus, D.Thilakawardana, R.Tafazolli\}@surrey.ac.uk.
}

\begin{abstract}
This paper investigates spectrum sharing (in the form of code sharing) between two Universal Mobile Telecommunication System (UMTS) operators in the UMTS extension band $(2500-2690 \mathrm{MHz})$ with equal and unequal number of proprietary carriers, respectively. The paper proposes a Dynamic Spectrum Allocation (DSA) algorithm to address the problem of spectrum sharing between two operators on a nonpool basis. It also investigates the impact of Adjacent Channel Interference (ACI) on the spectrum sharing gain. Additionally, an architecture that enables spectrum sharing to take place between two or more UMTS operators is presented. The simulated performance of the proposed DSA algorithm shows that under peak-hour loading, up to $32 \%$ increase in capacity can be obtained when compared to currently used Fixed Spectrum Allocation (FSA).
\end{abstract}

Keywords-Dynamic Spectrum Allocation (DSA); Fixed Spectrum Allocation (FSA).

\section{INTRODUCTION}

The electromagnetic spectrum is a scarce natural resource and needs to be utilized efficiently. The usage of the spectrum over the years has not been efficient. Though, there are many reasons why this is the case, the primary reason is the strong control of national and international regulatory bodies. The currently used static allocation mechanism is simple and provides interference protection between neighboring systems. It is however clearly inefficient and is inadequate in terms of innovations and rapid deployment of new technologies.

Technological advances in recent years and the exponential growth of communication systems has led to a rethinking of the way spectrum should be utilized. Currently, some bands of the radio spectrum are heavily congested whereas other bands are much less congested [1]. Also, studies have shown that at any given time and spatial location, the actual spectrum used is much less than the allocated spectrum [2]. Thus, there is a growing awareness that radio spectrum be shared as much as possible among the spectrum users without degrading their performance.

In realization of the growing importance of spectrum sharing, many regulatory bodies worldwide such as the Federal Communication Commission (FCC) in the USA and the Office of Communications (OFCOM) in the UK have indicated a policy shift towards flexible spectrum use [3-4]. Some past
European Union projects such as DRIVE [5] and OVERDRIVE [6], and some recent ones such as WINNER [7] and E2R [8] have also investigated solutions for flexible spectrum use among different Radio Access Technologies (RAT). Another demonstration of immense interest in this research field is the growing number of conferences such as CrownCom [9] and the IEEE DySpan [10] that address different themes of spectrum sharing.

The spectrum allocation for UMTS operators around $2 \mathrm{GHz}$ is already in place but for the new band $(2500-2690 \mathrm{MHz})$, the so called UMTS extension band, the allocation has not yet been made, and therefore it creates new exciting possibilities for spectrum sharing. This band can be shared either on a pool or non-pooled basis. The main difference between the two approaches is that in the pool based scenario, the resource is available to be shared jointly in a pool manner without prioritized access between the two operators. Hence, there is no notion of primary and secondary operators in pool based spectrum sharing. This work focuses on an algorithm for implementing the latter. Previous work in this area has examined partial cooperation between competing operators in the UMTS downlink [11]. This is within the context of nonshared radio access network, and it is also assumed that operators do not exchange operational information. Algorithms which limit the energy threshold by controlling the load have also been proposed [12]. However, architectural considerations necessary to enable UMTS spectrum sharing have not been addressed. This is addressed in this paper. Furthermore, it is commonly thought that during the busy hour, spectrum sharing is not possible between multiple operators of a RAT such as UMTS if they have equal number of carriers, as the busy hour is the same for all operators. However this does not imply that the peak of each operator's load during the busy hour is the same. This is exploited in this paper and is shown that when the peaks of the two operators during the busy hour are not same, a limited spectrum sharing is still possible if one of the operators has some spare capacity. Additionally, the operators may possess unequal number of carriers, so that even during the busy hour one may have enough capacity that could be utilised by another that needs capacity. This has also been investigated in this paper. The spectrum sharing gain in both of scenarios mentioned is as a 
result of the statistical multiplexing gain as well as the trunking gain. The statistical multiplexing gain is due to the nature of the call arrival while the trunking gain is primarily due to additional capacity offered by sharing the carrier. This paper considers a holistic approach to the problem of spectrum sharing between multiple UMTS operators. The performance of the proposed Dynamic Spectrum Allocation (DSA) algorithm is evaluated using a simulation tool and the results are compared with the Fixed Spectrum Allocation (FSA). In a deployed network scenario, the base stations of the different operators are physically separated. This causes inter-operator interference in the form of Adjacent Channel Interference $(\mathrm{ACI})$, in addition to the inter-cell and intra-cell interference. $\mathrm{ACI}$ reduces the available network capacity and hence the spectrum sharing gain. This paper models the impact of ACI on the results as well.

This work is divided into five main sections. Section two illustrates the spectrum sharing scenarios and presents a nonpool algorithm to enable spectrum sharing. Section three presents an architecture that enables spectrum sharing between multiple operators in UMTS. Section four describes the simulation tool and the algorithm performance in terms of the simulation results. Finally, some conclusions are drawn in section five of the paper.

\section{SCENARIOS AND ALGORITHM DESCRIPTION}

This section describes the scenarios that have been investigated in this paper. Before the description of the scenarios, it should be noted that spectrum sharing in UMTS refers to code sharing as UMTS is based upon the Wideband Code Division Multiple Access (WCDMA) technology. Some underlying assumptions are common to both scenarios investigated in this work and are described below.

In this spectrum sharing approach, there is a primary operator and a secondary operator. The operator that has spare capacity is termed as the primary operator, while the operator that suffers capacity crisis and requires additional spectrum is termed as the secondary operator. Both operators cover similar geographical area. They also share the Radio Network Controller (RNC) to address the issue of code management between the two operators and to share the DSA related information needed to co-ordinate the DSA process. The investigation is primarily for voice traffic in the present work. The effect of ACI is considered minimum when the base stations are co-located and maximum when the base stations are displaced [13]. Results for both cases are presented in this paper. Spectrum sharing on a non-pool basis can be further classified into two scenarios based upon the fact whether the sharing operators have equal or unequal number of carriers. The two cases considered are described next.

\section{A. Case I}

In this case, the two operators have similar traffic demand and same number of carriers, for example one carrier each. However there could be a relative difference in the peak hour loading between the primary and secondary operator that could be quite small to a relatively large value depending upon several factors such as market penetration, service quality and pricing of the operators. This variation can be exploited for the purpose of spectrum sharing. The main assumption is that the peak hours coincide, but the peak hour loading on the carriers of the two operators is different. The mechanism for spectrum sharing therefore requires a modification of the call admission block for the secondary operator, in a way that allows the operator to support its users on the primary network during capacity limited situations.

\section{B. Case II}

In this case, the two operators have different number of carriers. For example, the primary system has two carriers and the secondary system has one carrier while the traffic pattern of the two networks is similar. This case also requires a modification of the call admission process, such that users dropped or blocked from the secondary operator can be supported on the primary system. However, there is a priority for the primary users in access to the primary system. When a blocked call on the secondary system is supported on the primary system, this call may be dropped if a new primary user requires access to the system.

\section{Algorithm Description}

In the algorithm, higher priority assigned to the primary user of the shared spectrum. It considers the case for both equal and unequal number of carriers. All the decisions related to resource usage (including code sharing) are done by the RNC. Initially the secondary system uses its own carrier to support its own user until there is a capacity crisis on its network. Based on the algorithm the users in the secondary network are then allocated codes to enable connectivity on the primary system. The primary network has its own users and priority is given to them to avoid degrading the Quality of Service (QoS) of the primary network. Beyond a certain threshold at high loads, the primary system is no longer able to accommodate users from the secondary system and hence the secondary operator's calls are blocked/ dropped. The flowchart is shown in Fig. 1.

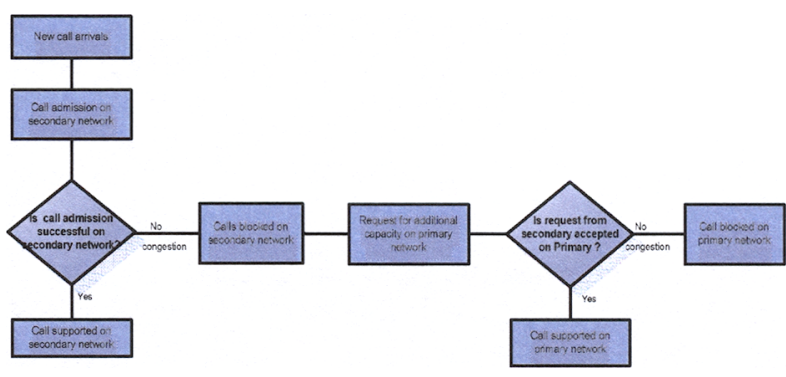

Figure 1 Flowchart of the spectrum sharing algorithm.

\section{SPECTRUM ACCESS ARCHITECTURE}

For the UMTS inter-operator sharing scenario, the sharing operators need to have some kind of co-ordination in the allocation of channelization / scrambling codes otherwise they will interfere with each other. Furthermore, issues like handover, power control, synchronization and channel 
estimation need to be revisited as new problems (e.g. how to do soft combining in case of handover if the base stations of the two operators are not co-located) will arise when operators share the spectrum. All these issues can be resolved if the operators are sharing the radio access network as well. The sharing of the access network is something at which 3GPP has thought about but not from the perspective of spectrum sharing but from the perspective of reducing the deployment and operational / maintenance cost of UMTS networks [14]. Their view of multiple operators sharing the radio access network is shown in [15-16]. The authors have extended this further to cover the scenario in which two operators are sharing the carriers (in the form of code sharing), either in a pool or nonpool manner. The entities which allow spectrum sharing to take place at the RNC have been defined in Fig. 2. The DSA Node is responsible for coordinating all DSA related activities at predefined intervals. It has spectrum knowledge and also collects loading information from the base stations. The Accounting Authorization and Authentication (AAA) platform is responsible for managing customer billing. Security management entity ensures that the sharing process is secured in terms of operator and user information. Code allocation entity dynamically allocates the codes and keeps track of code utilization in the shared system.

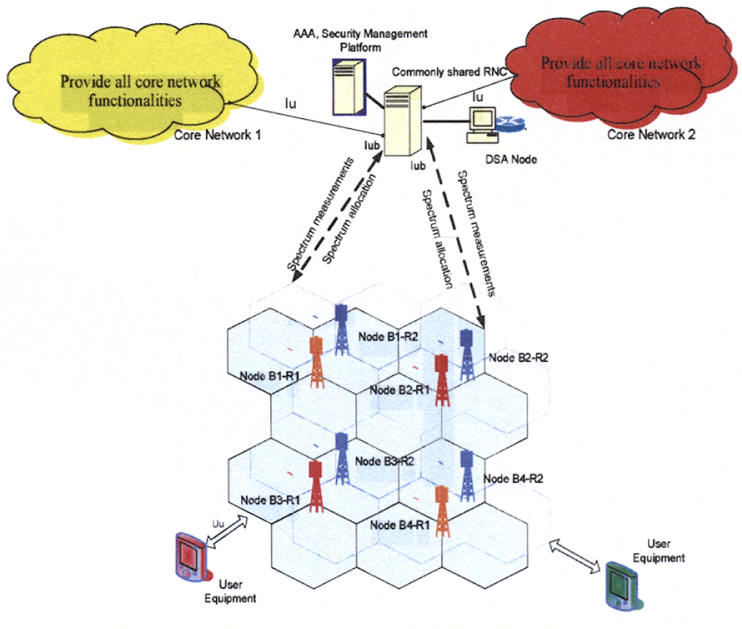

Figure 2 Spectrum access architecture.

\section{SYSTEM LEVEL DSA PROTOCOL PERFORMANCE AND PERFORMANCE}

A multi-operator system level simulator has been developed, in order to evaluate the performance of the DSA algorithm proposed. This system level simulator comprises of a number of modules shown in Fig. 3. The traffic module generates the relevant traffic information for the simulation. The users are assumed to be uniformly distributed over the coverage area. The mobile station module generates all mobile associated properties including the mobile ID, location, call arrival time, departure time and handover time. The simulation tool takes into account realistic traffic models [17]. The call arrivals are Poisson distributed and the call duration exponentially distribution [18]. The cell grid module generates the radio cells for multiple UMTS operators. Wraparound mobility model is used for interference calculation and to maintain a constant user density in the simulation. The interference model considers the distribution of users in the first tier cells. ACI which is due to imperfection in the transmitting and receiving filter characteristics is modeled according to [14]. The network control module is the core unit which connects to all the other parts of the simulator and also handles call admission for different operators. The Graphic User Interface (GUI) module is the user interface of the simulator and the spectrum module provides the various spectrum bands available for spectrum sharing.

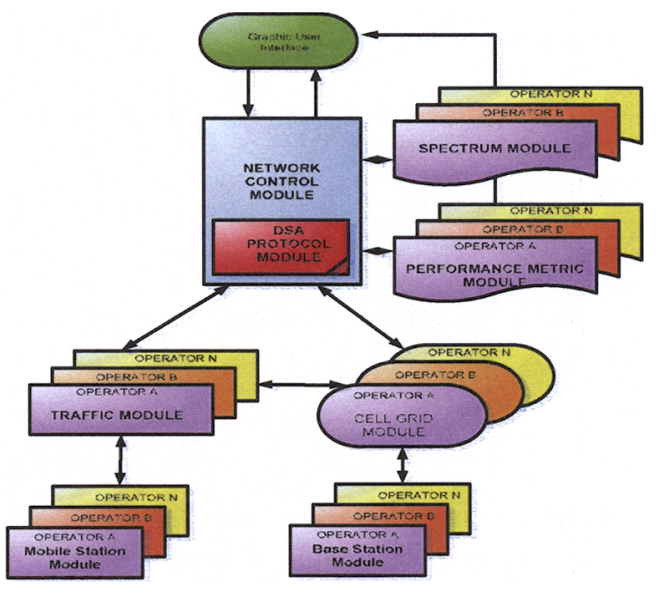

Figure 3 Multi-operator simulator block diagram.

\section{A. Simulation Results}

The important simulation parameters used in this work are shown in Table 1.

TABLE I. SIMULATION PARAMETERS

\begin{tabular}{|l|l|}
\hline \multicolumn{1}{|c|}{ Parameters } & \multicolumn{1}{c|}{ Values } \\
\hline Service type & Speech traffic \\
\hline Data rate & $12.2 \mathrm{Kbps}$ \\
\hline Call Duration & Mean 120 seconds ( Exponential) \\
\hline $\mathrm{E}_{\mathrm{b}} / \mathrm{N}_{\mathrm{o}}$ & $7 \mathrm{~dB}$ \\
\hline Adjacent Channel Interference & $2 \%$ \\
\hline Soft handover Gain & 3 \\
\hline Cell radius & $1 \mathrm{Km}$ \\
\hline Voice Activity Factor & 0.67 \\
\hline UMTS carrier bandwidth & $5 \mathrm{MHz}$ \\
\hline Chip rate & $3.84 \mathrm{MCps}$ \\
\hline Simulation borders & $\begin{array}{l}\text { Wraparound mobility of MS at } \\
\text { simulation borders }\end{array}$ \\
\hline Propagation Model & $\begin{array}{l}\text { Path loss model with } 4^{\text {th }} \text { order power } \\
\text { exponent }\end{array}$ \\
\hline User distribution & Uniform ( No active hotspots ) \\
\hline Frequency re-use factor & 1 \\
\hline Handover & Based on geometric cell boundaries \\
\hline Total Number of cells & 12 (with interference modeling ) \\
\hline Carrier distribution & $\begin{array}{l}\text { Primary ( } 1 \text { or 2 carriers ), secondary ( 1 } \\
\text { carrier) }\end{array}$ \\
\hline Cell layout & $\begin{array}{l}\text { Hexagonal with omni-directional antenna } \\
\text { deployment }\end{array}$ \\
\hline
\end{tabular}


In order to compare the performance of the DSA algorithm, the FSA curve has been obtained using the parameter specified in Table 1. The curve is shown in Fig. 4.

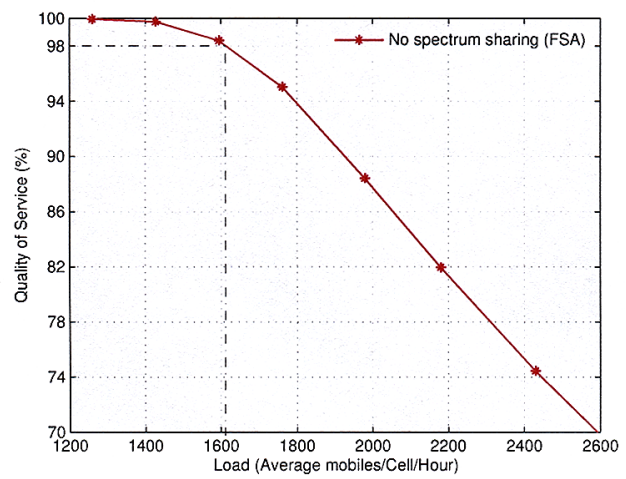

Figure 4 Reference FSA curve for comparing the performance of the DSA algorithm.

The horizontal axis in Fig. 4 represents the average arrival rate, i.e. number of the mobile users per cell in one hour (offered traffic / system load) and the vertical-axis represents Quality of Service (QoS) that defines the level of satisfaction received by the mobile users. This is defined as shown in (1).

QoS $=\frac{\text { Arrived Calls }- \text { Blocked Calls }- \text { Dropped Calls }}{\text { Arrived Calls }}$

The higher the number of mobile subscribers that can be accommodated in a cell, the higher the spectrum efficiency. The DSA gain $(\Delta \eta)$ formula is shown in (2). The increase in spectrum efficiency brought about by DSA over FSA is measured in terms of the additional load that is supported by the DSA algorithm relative to the load supported by the FSA algorithm at $98 \%$ satisfaction ratio. This value is considered sufficient to provide the desired QoS in an operator's network.

$$
\Delta \eta=\frac{\operatorname{Load}_{D S A}, 98 \%-\text { Load }_{F S A, 98 \%}}{\text { Load }_{F S A, 98 \%}}
$$

Load $_{D S A}$ 98\% Users/Cell/Hour for DSA at $98 \%$ Satisfaction ratio Load $_{F S A}, 98 \%$ Users/Cell/Hour for FSA at $98 \%$ Satisfaction ratio

\section{B. Case I}

The peak load on the primary system is mismatched by $5 \%, 10 \%, 15 \%$ and $20 \%$ respectively compared to the secondary system. Hence these additional capacities are available for sharing with the secondary system. This is shown in Table II. By implementing the spectrum sharing algorithm of Fig. 1 in the system level simulator, the results of the scenario (with mismatched loading on primary and secondary operators' carriers) are illustrated in Table III. It was confirmed that the spectrum sharing gain on the secondary system increased by an equal amount. It should be noted that the values of spare capacity in the primary was chosen to illustrate the concept, in real networks during the busy hour, the available capacity on the primary system could have a different value. In principle whatever capacity is available on the primary operator carrier could be utilized by the secondary operator if it requires capacity. As due to statistical nature of the arrival of traffic, it is unlikely that both operators will have exactly the same demand or same peak loading for all the time.

TABLE II. LOAD VARIATION BETWEEN PRIMARY AND SECONDARY SYSTEM (CASE I)

\begin{tabular}{|c|c|c|}
\hline $\begin{array}{c}\text { Loading of Secondary } \\
\text { system before sharing } \\
\text { (MS/Cell /Hour) }\end{array}$ & $\begin{array}{c}\text { Loading of Primary } \\
\text { system before sharing } \\
\text { (MS/Cell/Hour) }\end{array}$ & $\begin{array}{c}\text { Load variation } \\
(\%)\end{array}$ \\
\hline 1200 & 1140 & $5 \%$ \\
\hline 1200 & 1080 & $10 \%$ \\
\hline 1200 & 1020 & $15 \%$ \\
\hline 1200 & 960 & $20 \%$ \\
\hline
\end{tabular}

TABLE III. SPECTRUM SHARING GAIN ON SECONDARY SYSTEM (CASE I)

\begin{tabular}{|c|c|c|}
\hline $\begin{array}{c}\text { Loading of Secondary } \\
\text { system after sharing } \\
\text { (MS/Cell /Hour) }\end{array}$ & $\begin{array}{c}\text { Loading of Primary } \\
\text { system after sharing } \\
\text { (MS/Cell/Hour) }\end{array}$ & $\begin{array}{c}\text { Gain from } \\
\text { sharing (\%) }\end{array}$ \\
\hline 1260 & 1140 & $5 \%$ \\
\hline 1320 & 1080 & $10 \%$ \\
\hline 1380 & 1020 & $15 \%$ \\
\hline 1440 & 960 & $20 \%$ \\
\hline
\end{tabular}

\section{Case II}

Fig. 5 and Fig. 6 show the result of the algorithm with unequal number of carriers. The DSA curve of the secondary operator shows improvement in capacity until a point, after which it starts to decrease. This initial increase is due to the additional capacity provided by the primary operator. It is observed that there is a point on the DSA curve after which no further increase in the gain is obtained. This point represents the saturation point of the secondary users on the primary system. This is because the algorithm allows the primary users to have priority. In the case of competition between primary and secondary users, access is granted to the primary users and the secondary user is blocked. The gain performance shows a $36 \%$ and $32 \%$ increase in capacity respectively without and with ACI. The $4 \%$ loss in capacity is due to ACI. The DSA performance approaches the FSA performance at high load since the primary operator requires its own carrier to support the incoming primary users. 


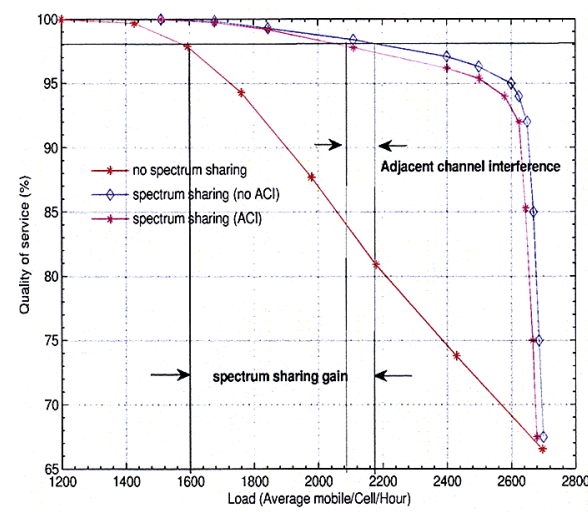

Figure 5 Spectrum sharing gain for secondary system (Case II)

Fig. 6 also shows that there is no harmful degradation to the primary system due to the priority of the primary users over secondary users.

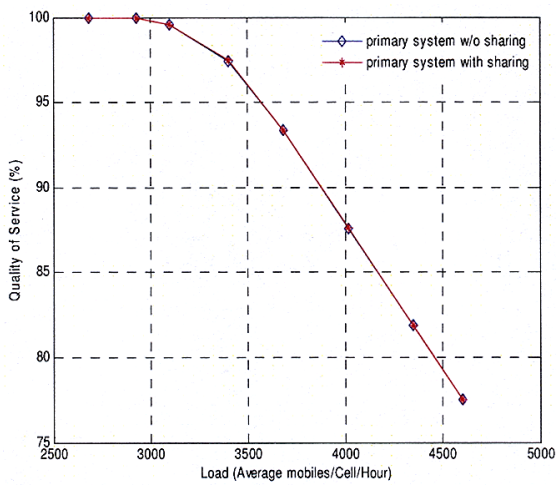

Figure 6 Performance of primary system when sharing spectrum with the secondary system (Case II).

\section{CONCLUSIONS}

Based on initial investigations, the two cases of the nonpool DSA algorithm have been presented. It can be seen that spectrum sharing between two operators with equal number is possible using this approach. The results show that even during the busy hour, any available capacity on the primary operator could be utilized for spectrum sharing purposes if the secondary operator is in need of additional capacity. For unequal number of carriers, the spectrum sharing gain is primarily due to additional spectrum available for sharing. It was also observed that secondary users can be accommodated on the primary system without degrading the primary system performance since the algorithm ensures adequate protection to the primary users through prioritization. Furthermore, the access architecture to facilitate sharing between two operators has also been presented. The simulation results also show that Adjacent Channel interference (ACI) reduces the spectrum sharing gain up to $4 \%$ in the scenarios investigated. A spectrum sharing gain of $32 \%$ (with $\mathrm{ACI}$ ) has been obtained with unequal number of carriers' scenario, due to increased ability of the secondary system to accommodate more mobile subscribers through spectrum sharing with the primary.

\section{ACKNOWLEDGMENT}

The work reported in this paper has formed part of the Delivery Efficiency Core Research Programme of the Virtual Centre of Excellence in Mobile \& Personal Communications, Mobile VCE, www.mobilevce.com. This research has been funded by the Industrial Companies who are Members of Mobile VCE, with additional financial support from the UK Government's Engineering \& Physical Sciences Research Council. Fully detailed technical reports on this research are available to Industrial Members of Mobile VCE.

\section{REFERENCES}

[1] Shared Spectrum Company website: http://www.sharedspectrum.com/measurements/recent.html [Accessed 11th February 2008]

[2] Office of Communication, "Technology research programme," Research and development at Ofcom , 2005/06.

[3] FCC 04-167, " Promoting efficient use of spectrum through elimination of barriers to the development of secondary markets," Jan. 2004.

[4] Office of communication (Ofcom), "Spectrum Usage Rights: Technology and Usage Neutral Access to the Spectrum", 2006.

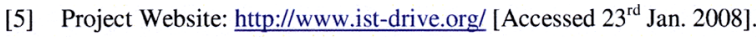

[6] Project Website:http://www.comnets.rwth-aahen.de/ o_drive/ [Accessed $23^{\text {rd }}$ January 2008].

[7] Project Website: https://www.ist-winner.org/ [Accessed 8th Nov. 2007]

[8] Project Website: http://e2r2.motlabs.com/ [Accessed 16 ${ }^{\text {th }}$ Jan. 2008].

[9] International Conference on Cognitive Radio Oriented Wireless Networks and Communications website: http://www.crowncom.org/ [Accessed $20^{\text {th }}$ December 2007].

[10] IEEE Dynamic Spectrum Access Networks, conference website: http://www.ieee-dyspan.com/ [Accessed $18^{\text {th }}$ Jan. 2008].

[11] M.K. Pereirasamy, J. Luo, M. Dillinger, C. Hartmann, "Inter-operator spectrum sharing for UMTS FDD," Proceeding of the $5^{\text {th }}$ World Wireless Congress (WWC), San Francisco, USA, May 2004.

[12] M.K. Pereirasamy, J.Luo, M. Dillinger, C.Hartmann, "An Approach for Inter-Operator Spectrum Sharing for 3G Systems and Beyond," Proceedings of $15^{\text {th }}$ IEEE Int. Symp. On Personal, Indoor and Mobile radio Communications (PIMRC), Barcelona, Spain, Sept 2004.

[13] $3^{\text {rd }}$ Generation Partnership Project, Technical Specification Group Radio Access Networks; TR 25.942 v5.1.0, RF System Scenarios (Release 5), June 2002.

[14] H. Holma, A. Toskala, WCDMA for UMTS Radio Access for third generation Mobile Communication, John Wiley \& Sons, 2000.

[15] $3^{\text {rd }}$ Generation Partnership Project, Technical Specification Group Services and Systems Aspects; TS 23.251 v6.6.0, Network Sharing; Architecture and functional description (Release 6), March 2006.

[16] J. Laiho, A. Walker, T.Novosad, Radio Network Planning and Optimisation, John Wiley and Sons, 2002.

[17] J. Guo, F. Liu, Z. Zhu, "Estimate the Call Duration Parameters in GSM System Based on K-L Divergence Method," Proceedings of International Conference on Wireless Communications, Networking and Mobile Computing, 21-25 Sept. 2007.

[18] W.H. Tranter, K.S. Shanmugan, T.S Rappaport, K.L. Kosbar, Principles of Communication Systems Simulation with Wireless Applications, Pearson Education, 2004. 